\title{
26 Research Soure \\ Development Of A Scoring System With \\ Multidimensional Markers For Fibrosing Interstitial \\ Lung Disease
}

\section{Shenyun Shi}

Nanjing Drum Tower Hospital, Clinical College of Nanjing Medical University

\section{Lulu Chen}

Nanjing Drum Tower Hospital, Clinical College of Nanjing Medical University

\section{Xiaoqin Liu}

Nanjing Drum Tower Hospital, the Affiliated Hospital of Nanjing University Medical School

Min Yu

Nanjing Drum Tower Hospital, Clinical College of Nanjing Medical University

Chao Wu

Nanjing Drum Tower Hospital, Clinical College of Nanjing Medical University

Yonglong Xiao ( $\nabla$ yonglong11a@163.com )

Nanjing Drum Tower Hospital, Clinical College of Nanjing Medical University

\section{Research Article}

Keywords: interstitial lung disease, connective tissue disease, idiopathic pulmonary fibrosis, prognosis, scoring system, biomarkers

Posted Date: March 7th, 2022

DOI: https://doi.org/10.21203/rs.3.rs-1414607/v1

License: (9) This work is licensed under a Creative Commons Attribution 4.0 International License.

Read Full License 


\section{Abstract}

Fibrosing interstitial lung disease (ILD) can cause high mortality and sensitive evaluation of fibrosing ILD could be critical. The aim of this study is to develop a scoring system to predict prognosis of fibrosing ILD. We retrospectively reviewed 339 fibrosing ILD patients admitted to Nanjing Drum Tower Hospital from February 2017 to February 2020. All patients were followed up until September 2020. Cox multiple regression analysis indicated that smoking history $(\mathrm{HR}=3.826$, $\mathrm{P}=0.001)$, age $(\mathrm{HR}=1.043, \mathrm{P}=0.015), \mathrm{CEA}(\mathrm{HR}=1.059, \mathrm{P}=0.049)$,CYFRA21-1 $(\mathrm{HR}=1.177, \mathrm{P}=0.004)$ and $\mathrm{DLCO} \%$ predicted $(H R=0.979, P=0.032)$ were independent prognostic factors for fibrosing ILD. The clinical scoring system for fibrosing ILD was established based on the clinical variables (age [A], CEA and CYFRA21-1 [C], DLCO\% predicted [D], and smoking history [S]; ACDS). The area under the receiver operating characteristic curve (AUROC) of the scoring system for predicting prognosis of fibrosing ILD was 0.91 ( $95 \% \mathrm{Cl}$ : 0.87 $0.94, \mathrm{P}<0.001)$. The cutoff value was 2.5 with their corresponding specificity $(91.1 \%)$ and sensitivity (77.5\%). Correlation analyses showed that the increased age, CEA and CYFRA21-1 were significantly correlated with decreased DLCO\%predicted $(P<0.001, P=0.028, P<0.001$, respectively). The independent risk factors for mortality in patients with fibrosing ILD are higher CEA, higher CYFRA21-1, smoking history, lower DLCO\%predicted at baseline and older age. ACDS is a simple and feasible clinical model for predicting survival of fibrosing ILD.

\section{Background}

Interstitial lung diseases (ILDs) are a group of heterogeneous lung diseases with pulmonary alveolar unit inflammation or interstitial fibrosis that are associated with substantial morbidity and mortality. The causation of ILD includes idiopathic and specific etiology including autoimmune disease, vasculitis, drugs, tumors and occupational or environmental exposure(1-2). Idiopathic pulmonary fibrosis (IPF) is one of a family of idiopathic interstitial pneumonias characterized by usual interstitial pneumonia (UIP) in high-resolution computed tomography (HRCT) and pathology(3). IPF has a poor prognosis with median survival from the time of diagnosis approximately 3 years(4). Fibrosing ILDs other than IPF, such as connective tissue disease (CTD) associated ILD, including ILD associated with rheumatoid arthritis (RA-ILD), systemic sclerosis (SSc-ILD) and polymyositis/dermatomyositis are also known to have progressive disease behaviors similar to IPF(5-7). Therefore, it is important to recognize the risk factors associated with poor prognosis in patients with fibrosing interstitial lung disease.

Several biomakers has been reported to be as diagnostic and prognostic biomarkers of fibrosing ILD, including Krebs von den lugen-6 (KL-6), Surfactant proteins A and D (SP-A and SP-D), serum interleukin 6 (IL-6) levels and tumor markers(8-10). However, the relationship between proportion of each serum marker and fibrosing ILD is not clear. Therefore, in this study, we retrospectively studied the clinical characteristics of patients with fibrosing ILD and established a novel model to better guide personalized therapeutic choices in persons.

\section{Materials And Methods}




\subsection{Study subjects}

We retrospectively reviewed 339 patients who were newly diagnosed of fibrosing ILD (IPF and CTD-ILD) from inpatient of the department of respiration of Nanjing Drum Tower Hospital from February 2017 to February 2020(Figure 1). Patients with combined pneumonia, pulmonary tuberculosis, lung malignancy, or other pulmonary diseases were excluded. The follow-up period was from 7 months to 3 years. We analyzed demographic features, clinical characteristics, lung function parameters and therapy.

This study was consented by Ethics Committee of Nanjing Drum Tower Hospital. The Ethics Committee waived the need for informed consent as the study was retrospective and the data were analyzed anonymously.

\subsection{Methods}

The diagnosis for IPF was mainly based on the criteria from An Official ATS/ERS/JRS/ALAT Clinical Practice Guideline (3). The diagnosis of CTD-ILD referred to the published guideline (11). Clinical information at admission was collected including demographics, smoking history. Pulmonary function tests including forced vital capacity (FVC), FVC\% predicted, diffusion capacity for carbon monoxide (DLCO), and DLCO\% predicted were extracted for analysis. All subjects had UIP pattern on chest HRCT as defined by the guidelines from the American thoracic society and the European respiratory society $(3,11)$.

\subsection{Statistical analysis}

Data were expressed as mean \pm standard deviation (SD). t-Test or the Mann-Whitney $U$ test was used for continuous variables. Categorical variables were compared by Chi-square test. The independent prognostic role of variables were evaluated by Cox proportional hazard analysis. Receiver operator characteristic (ROC) analyses were performed to calculate area under the ROC curve (AUC) of markers for predicting the prognosis of fibrosing ILD. Pearson correlation analysis was used to evaluate the relationship between markers and lung function parameters. All analyses were performed with SPSS software, version 23.0 and GraphPad Prism version 7 (Graph Pad Software Inc., La Jolla, CA, USA). Statistical significance was considered at the 0.05 levels.

\section{Results}

\subsection{Baseline clinical characteristics of fibrosing ILD patients}

The baseline clinical features of subjects with IPF $(n=132)$ and CTD-ILD characterized by UIP on HRCT $(n=207)$ were summarized in Table 1. Female gender, older age were more common in the IPF group ( $p<$ 0.001 and $p<0.001$, respectively). Smoking history was similar. Red blood cell distribution width(RDW) levels, serum total bilirubin (TBIL) and direct bilirubin (DBIL) also differed between the two groups $(p=$ $0.008, p<0.001$ and $p=0.001$, respectively). Patients with CTD-ILD characterized by UIP on HRCT had a higher DLCO\% predicted level compared with the IPF patients. 
Table 1

Baseline clinical features

\begin{tabular}{|c|c|c|c|c|}
\hline Variables & UIP( $(n=339)$ & $\operatorname{IPF}(n=132)$ & CTD-UIP( $(n=207)$ & $p$ value \\
\hline Gender (M/F) & $183 / 156$ & $115 / 17$ & $68 / 139$ & $<0.001$ \\
\hline Smoking history $(\mathrm{Y} / \mathrm{N})$ & $60 / 279$ & $31 / 101$ & $29 / 178$ & 0.026 \\
\hline Age (years old) & $62.49 \pm 11.24$ & $68.27 \pm 8.71$ & $58.80 \pm 11.13$ & $<0.001$ \\
\hline WBC count $\left(\times 10^{\wedge} 9\right)$ & $7.01 \pm 2.07$ & $7.11 \pm 1.91$ & $6.94 \pm 2.16$ & 0.46 \\
\hline RDW(\%) & $13.63 \pm 1.25$ & $13.42 \pm 1.03$ & $13.76 \pm 1.35$ & 0.008 \\
\hline $\operatorname{PLT}\left(\times 10^{\wedge} 9\right)$ & $211.68 \pm 71.57$ & $190.62 \pm 73.37$ & $225.11 \pm 67.21$ & $<0.001$ \\
\hline TBil(umol/l) & $8.99 \pm 3.28$ & $9.81 \pm 3.32$ & $8.48 \pm 3.16$ & $<0.001$ \\
\hline DBil(umol/l) & $2.68 \pm 1.22$ & $2.96 \pm 1.23$ & $2.50 \pm 1.18$ & 0.001 \\
\hline $\mathrm{LDH}(\mathrm{U} / \mathrm{L})$ & $265.18 \pm 77.13$ & $254.52 \pm 63.34$ & $271.98 \pm 84.20$ & 0.031 \\
\hline B cells $\left(\times 10^{\wedge} 9\right)$ & $0.237 \pm 0.161$ & $0.244 \pm 0.148$ & $0.233 \pm 0.169$ & 0.550 \\
\hline NK cells $\left(\times 10^{\wedge} 9\right)$ & $0.263 \pm 0.197$ & $0.329 \pm 0.229$ & $0.222 \pm 0.160$ & $<0.001$ \\
\hline CEA $(\mathrm{ng} / \mathrm{ml})$ & $2.61 \pm 2.89$ & $3.20 \pm 2.21$ & $2.24 \pm 3.17$ & 0.003 \\
\hline CYFRA21-1 (ng/ml) & $4.36 \pm 2.14$ & $4.58 \pm 2.04$ & $4.22 \pm 2.19$ & 0.131 \\
\hline NSE (ng/ml) & $16.58 \pm 5.56$ & $16.69 \pm 6.74$ & $16.51 \pm 4.68$ & 0.772 \\
\hline $\mathrm{PaO} 2 / \mathrm{FiO} 2$ ratio & $367.20 \pm 75.55$ & $370.36 \pm 86.27$ & $364.76 \pm 66.32$ & 0.551 \\
\hline FVC\% predicted & $67.10 \pm 17.29$ & $66.72 \pm 17.17$ & $67.35 \pm 17.41$ & 0.746 \\
\hline FEV1\% predicted & $73.50 \pm 18.44$ & $73.25 \pm 17.37$ & $73.65 \pm 19.13$ & 0.847 \\
\hline DLCO\% predicted & $52.69 \pm 21.43$ & $49.47 \pm 19.02$ & $54.74 \pm 22.32$ & 0.027 \\
\hline
\end{tabular}

\subsection{Constructing a scoring system for predicting prognosis of fibrosing ILD}

According to the final follow-up data, 339 fibrosing ILD patients were divided into survivors group ( $\mathrm{n}=$ 259 ) and decedents group $(n=80)$. Cox proportional hazards models were used to examine the influence of variables on the prognosis of patients with fibrosing ILD. The multivariate cox regression analysis showed that smoking history $(H R=3.826, P=0.001)$, age $(H R=1.043, P=0.015)$, carcinoem-bryonic antigen $(C E A)(H R=1.059, P=0.049)$, cytokeratin $21-1(C Y F R A 21-1)(H R=1.177, P=0.004)$ and DLCO\%predicted $(H R=0.979, P=0.032)$ were independent prognostic factors for fibrosing ILD(Table 2$)$. 
Table 2

Prognostic factors for survival by univariate and multivariate Cox regression models in fibrosing ILD patients

\begin{tabular}{|lllllll|}
\hline Variables & \multicolumn{3}{l}{ Univariate Cox Model } & \multicolumn{4}{l|}{ Multivariate Cox Model } \\
\cline { 2 - 7 } & HR & $95.0 \%$ Cl & p value & HR & $95.0 \%$ Cl & p value \\
\hline CTD-UIP(Y) & 2.234 & $1.428-3.496$ & $<0.001$ & 1.857 & $0.835-4.134$ & 0.129 \\
\hline Gender & 1.376 & $0.883-2.144$ & 0.159 & 0.369 & $0.133-1.024$ & 0.056 \\
\hline Smoking history & 5.096 & $3.279-7.920$ & $<0.001$ & 3.826 & $1.686-8.683$ & 0.001 \\
\hline Age (years old) & 1.069 & $1.045-1.093$ & $<0.001$ & 1.043 & $1.008-1.079$ & 0.015 \\
\hline WBC count & 1.163 & $1.054-1.282$ & 0.003 & 1.008 & $0.843-1.206$ & 0.926 \\
\hline RDW & 1.391 & $1.216-1.591$ & $<0.001$ & 1.075 & $0.837-1.381$ & 0.572 \\
\hline PLT & 1.000 & $0.997-1.003$ & 0.765 & 0.999 & $0.994-1.003$ & 0.591 \\
\hline TBil & 0.964 & $0.897-1.035$ & 0.310 & 0.890 & $0.775-1.023$ & 0.101 \\
\hline DBil & 1.015 & $0.853-1.207$ & 0.871 & 1.260 & $0.808-1.966$ & 0.308 \\
\hline LDH & 1.003 & $1.001-1.006$ & 0.002 & 1.002 & $0.997-1.006$ & 0.493 \\
\hline B cells & 0.710 & $0.180-2.797$ & 0.624 & 1.733 & $0.348-8.634$ & 0.502 \\
\hline NK cells & 1.652 & $0.572-4.772$ & 0.354 & 2.173 & $0.417-11.324$ & 0.357 \\
\hline CEA & 1.084 & $1.046-1.123$ & $<0.001$ & 1.059 & $1.000-1.122$ & 0.049 \\
\hline CYFRA21-1 & 1.374 & $1.290-1.463$ & $<0.001$ & 1.177 & $1.053-1.316$ & 0.004 \\
\hline NSE & 1.051 & $1.015-1.087$ & 0.004 & 1.015 & $0.970-1.061$ & 0.520 \\
\hline PaO2/FiO2 ratio & 0.993 & $0.990-0.997$ & $<0.001$ & 0.998 & $0.993-1.002$ & 0.302 \\
\hline FVC\% predicted & 0.955 & $0.940-0.970$ & $<0.001$ & 1.010 & $0.956-1.066$ & 0.732 \\
\hline FEV1\% predicted & 0.970 & $0.957-0.983$ & $<0.001$ & 0.965 & $0.918-1.015$ & 0.171 \\
\hline DLCO\% predicted & 0.949 & $0.937-0.960$ & $<0.001$ & 0.979 & $0.959-0.998$ & 0.032 \\
\hline
\end{tabular}

The accuracy of independent prognostic factors for predicting the survival of fibrosing ILD was then evaluated by Receiver Operating Characteristics (ROC) analysis. The area under the ROC curve for CYFRA21-1 in predicting the survival of fibrosing ILD was $0.85(95 \% \mathrm{Cl}, 0.80-0.90 ; p<0.001)$. The prediction ability for smoking history, age, CEA and DLCO\%predicted were listed in Table 3. Then, we constructed a simple clinical scoring system for predicting survival of fibrosing ILD with the variables of smoking history, age, CEA, CYFRA21-1 and DLCO\%predicted (Table 4). ROC curve was calculated to compare the predictive value of the scoring system. The ROC curve was shown in Fig. 2 . The area under 
the curve of the scoring system for predicting survival of fibrosing ILD was 0.91 (95\% Cl: $0.87-0.94, \mathrm{P}<$ $0.001)$. The cutoff value was 2.5 with their corresponding specificity $(91.1 \%)$ and sensitivity $(77.5 \%)$.

Table 3

Comparisons of ROC curve analysis for predicting the survival of fibrosing ILD patients

\begin{tabular}{|c|c|c|c|c|c|}
\hline & $\mathrm{AUC}(95 \% \mathrm{Cl})$ & $p$ value & Cut-off value & Sensitivity & Specificity \\
\hline Smoking history & $0.69(0.61,0.76)$ & $<0.001$ & - & - & - \\
\hline Age (years old) & $0.69(0.63,0.76)$ & $<0.001$ & 66.5years old & $64.60 \%$ & $66.30 \%$ \\
\hline CEA & $0.62(0.55,0.70)$ & $<0.001$ & $2.29 \mathrm{ng} / \mathrm{ml}$ & $60.80 \%$ & $61.60 \%$ \\
\hline CYFRA21-1 & $0.85(0.80,0.90)$ & $<0.001$ & $4.34 \mathrm{ng} / \mathrm{ml}$ & $88.60 \%$ & $74.40 \%$ \\
\hline DLCO\% predicted & $0.84(0.79,0.89)$ & $<0.001$ & $40.1 \%$ & $72.50 \%$ & $87.30 \%$ \\
\hline
\end{tabular}

Table 4

Development of a clinical scoring system to predict survival of interstitial pneumonia characterized by UIP in HRCT

\begin{tabular}{|llll|}
\hline Variables & & Cut-off value & Score \\
\hline A & & & \\
\hline & Age & $\leq 66.5$ years & 0 \\
\hline & & $>66.5 y e a r s$ & 1 \\
\hline C & CEA & $\leq 2.29 \mathrm{ng} / \mathrm{ml}$ & 0 \\
\hline & & $>2.29 \mathrm{ng} / \mathrm{ml}$ & 1 \\
\hline & CYFRA21-1 & $\leq 4.34 \mathrm{ng} / \mathrm{ml}$ & 0 \\
\hline & & $>4.34 \mathrm{ng} / \mathrm{ml}$ & 1 \\
\hline D & DLCO\% predicted & $>40.1 \%$ & 0 \\
\hline & & $\leq 40.1 \%$ & 1 \\
\hline S & Smoking history & No & 0 \\
\hline & & Yes & 1 \\
\hline
\end{tabular}

\subsection{Correlations between prognostic factors and lung function parameters}

Correlation analyses showed that DLCO\%predicted levels were negatively correlated with age $(r=-0.225, p$ $<0.001), \mathrm{CEA}(\mathrm{r}=-0.119, \mathrm{p}=0.028)$ and CYFRA21-1 $(\mathrm{r}=-0.292, \mathrm{p}<0.001)$ (Fig. 3). 


\section{Discussion}

The present study retrospectively compared the clinical and follow-up data between 259 survivors and 80 decedents with fibrosing ILD. In this study, we demonstrated that smoking history, age, CEA, CYFRA21-1 and DLCO\% predicted could predict the survival of fibrosing ILD patients independently. A new predictive scoring system namely ACDS (age [A], CEA and CYFRA21-1 [C], DLCO\% predicted [D], and smoking history [S]) was proposed. Importantly, we found that scoring system level was closely associated with the prognosis of fibrosing ILD patients. Furthermore, the increased age, CEA and CYFRA21-1 were positively associated with the severity of patients with fibrosing ILD.

Fibrosing ILD had similar biological and clinical behaviours which was characterised by progressive deterioration in lung function, progressive deterioration in lung function and high mortality rate(12-13). Investigating the prognostic value of markers across fibrosing ILD was of great importance to clinical evaluation and make continues to elucidate the approach to fibrosing ILD management. In the past few years, several serum markers were identified as simple and readily accessible biomarkers to predict the survival and severity of fibrosing ILD. There were researches studying tumor markers such as CEA, carbohydrate antigen $19-9$ (CA $19-9)$ and CYFRA21-1 that might reflect the severity and prognosis of fibrosing ILD(14-16). One retrospective study by Fahim A et al., which included 41 non-smoking patients with idiopathic pulmonary fibrosis(IPF), reported that serum CEA concentration was elevated in approximately half of patients with IPF and was correlated with disease severity(17). These results were consistent with the finding of our study of CEA as a biomarker in fibrosing ILD patients. In our study, CEA was identified as an independent prognostic factor for fibrosing ILD. CEA is a glycoprotein involved in cell adhesion and is produced by colonic epithelium. It has reported that CEA localizes in metaplastic epithelium lining honeycombed bronchioles by immunohistochemical staining. As cuboidal pneumocytes are the predominant source of epithelial renewal in severe lung damage and fibrosis, these cells are the most likely source of CEA release(18).

In this study, elevated serum levels of CYFRA21-1 were observed in decedents group with fibrosing ILD. In a study by Vercauteren et al., higher level of CYFRA 21 - 1 in BAL of IPF patients resulted in worse survival in comparison with the CYFRA 21 - 1 low counterpart (19). The expression of CYFRA21-1 in the lung has been identified in bronchiolar epithelial cells and pneumocytes. Elevation of serum CYFRA21-1 concentration might be associated with lysis or regeneration of these cells (15). Furthermore, we demonstrated that serum CEA and CYFRA21-1 were significantly correlated with decreased DLCO\%predicted in this study. The severity of ILD is usually based on pulmonary function test results such as DLCO\%predicted(20). Thus, serum CEA and CYFRA21-1 levels might be useful for reflecting the severity of fibrosing ILD.

A large amount of studies reported that smoking was closely associated with the onset and progress of pulmonary fibrosis(21-22). A possible explanation may be that cigarettes contain the cytotoxic, mutagenic and proinflammatory substances. According to previous reports, these substances caused cellular oxidative stress, increased epithelial cell apoptosis, and dysregulation of immune responses, 
which was responsible for the progress of pulmonary fibrosis(23-24). In addition, smoking affects the function of macrophages. It induced macrophage polarization to M2 phenotype that enhance the regression of inflammation and tissue remodeling(25). Therefore, smoking cessation could be a good way to slow down the development of pulmonary fibrosis in the patients with ILD.

In the past years, few models has been proposed to predict the severity and prognosis of IPF. Glasgow prognostic score (GPS) has been reported to play an important role in predicting mortality in patients with acute exacerbation of IPF(26). In our study, smoking history, age, CEA, CYFRA21-1 and DLCO\% predicted were identified as independent factors for predicting the prognosis of fibrosing ILD. Moreover, based on these variables, a new predictive scoring system namely ACDS (age [A], CEA and CYFRA21-1 [C], DLCO\% predicted [D], and smoking history [S]) was proposed. The scoring system was demonstrated to be as a predictive value for the survival of fibrosing ILD. Nevertheless, serum CEA and CYFRA21-1 levels might be useful for reflecting the severity of fibrosing ILD. However, it still needs further perspective study to verify the power of this scoring system based on multicenter and large population of fibrosing ILD patients.

Some limitations of this study should be noted. First, this was a retrospective and observational study of data obtained from a single center. In addition, the mechanism underlying the association of each biomarker with fibrosing ILD remains to be clarified in further in vivo and in vitro studies.

\section{Conclusions}

In conclusion, smoking history, age, CEA, CYFRA21-1 and DLCO\% predicted were independent predictors of the prognosis of fibrosing ILD patients that offers the advantages of convenience, ease of accessibility and low cost. A new predictive scoring system namely ACDS may help predict prognosis in patients with fibrosing ILD. Serum CEA and CYFRA21-1 levels might be useful for reflecting the severity of fibrosing ILD.

\section{Declarations}

\section{Ethics approval and consent to participate}

The study was conducted according to the Declaration of Helsinki. This study was approved by Ethics Committee of Nanjing Drum Tower Hospital, the Affiliated Hospital of Nanjing University Medical School.The Ethics Committee of Nanjing Drum Tower Hospital, the Affiliated Hospital of Nanjing University Medical School waived the need for informed consent as the study was retrospective and the data were analyzed anonymously.

\section{Consent for publication}

Not applicable.

\section{Availability of data and material}


The datasets used and/or analysed during the current study available from the corresponding author on reasonable request.

\section{Competing Interests}

All authors declare that there is no conflict of interests regarding the publication of this paper.

\section{Conflicts of Interest}

The authors declare that there is neither conflict of interest nor financial gain regarding the publication of this paper.

\section{Funding statement}

None declared.

\section{Authors' contributions}

S-Y S, L-L C, X-Q L, C W, M Y and Y-L X were involved in conception and design. S-Y S, L-L C, X-Q L and M $Y$ were involved in analysis and interpretation. S-Y S, and L-L $C$ were involved in acquisition of data. S-Y S, $X-Q, L, M ~ Y$ and $Y-L X$ were involved in writing and revisions.

\section{Acknowledgments}

Not applicable.

\section{References}

1. Antoniou K. M, Margaritopoulos G. A,Tomassetti S, Bonella F, Costabel U,Poletti V. Interstitial lung disease. Eur Respir Rev 23, 40-54 (2014).

2. Ito Y, Arita M, Kumagai S, Takei R, Noyama M, Tokioka F,Nishimura K, Koyama T, Notohara K, Ishida T. Serological and morphological prognostic factors in patients with interstitial pneumonia with autoimmune features. BMC Pulm Med 17, 111 (2017).

3. Raghu G, Remy-Jardin M, Myers JL, et al. Diagnosis of idiopathic pulmonary fibrosis. An official ATS/ ERS/JRS/ALAT clinical practice guideline. Am J Respir Crit Care Med 198, e44-e68 (2018).

4. Nicholson AC, Colby TV, Dubois RM, et al. The prognostic significance of the histologic pattern of interstitial pneumonia in patients presenting with the clinical entity of cryptogenic fibrosing alveolitis. American Journal of Respiratory and Critical Care Medicine 162, 2213-2217 (2000).

5. Zamora-Legoff JA, Krause ML, Crowson CS, et al. Progressive decline of lung function in rheumatoid arthritis-associated interstitial lung disease. Arthritis Rheumatol 69, 542-549 (2017).

6. Guler SA, Winstone TA, Murphy D, et al. Does systemic sclerosis-associated interstitial lung disease burn out? Specific phenotypes of disease progression. Ann Am Thorac Soc 15, 1427-1433 (2018). 
7. Marie I, Hatron PY, Dominique S, et al. Short-term and long-term outcomes of interstitial lung disease in polymyositis and dermatomyositis: a series of 107 patients. Arthritis Rheum 63, 439-3447 (2011).

8. Hamai K, Iwamoto H, Ishikawa N, et al. Comparative study of circulating MMP-7, CCL18, KL-6, SP-A, and SP-D as disease markers of idiopathic pulmonary fibrosis. Dis Markers 2016, 4759040 (2016).

9. De Lauretis A, Sestini P, Pantelidis P, et al. Serum interleukin 6 is predictive of early functional decline and mortality in interstitial lung disease associated with systemic sclerosis. J Rheumatol 40, 435446 (2013).

10. Maher TM, Oballa E, Simpson JK, et al. An epithelial biomarker signature for idiopathic pulmonary fibrosis: an analysis from the multicentre PROFILE cohort study. Lancet Respir Med 5, 946-955 (2017).

11. Travis WD, Costabel U, Hansell DM, et al. An official American thoracic society/European respiratory society statement: update of the international multidisciplinary classification of the idiopathic interstitial pneumonias. American Journal of Respiratory and Critical Care Medicine 188, 733-748 (2013).

12. Flaherty KR, Brown KK, Wells AU, et al. Design of the PF-ILD trial: a double-blind, randomised, placebo-controlled phase III trial of nintedanib in patients with progressive fibrosing interstitial lung disease. BMJ Open Respir Res 4, e000212 (2017).

13. Wells AU, Brown KK, Flaherty KR, et al. What's in a name? That which we call IPF, by any other name would act the same. Eur Respir J 51, 1800692 (2018).

14. Fujita J, Obayashi Y, Yamadori I, et al. Marked elevation of CA19-9 in a patient with idiopathic pulmonary fibrosis: CA19-9 as a bad prognostic factor. Respirology 3, 211-214 (1998).

15. Kanazawa $H$, Yoshikawa T, Yamada M, et al. CYFRA 21 - 1, a cytokeratin subunit 19 fragment, in bronchoalveolar lavage fluid from patients with interstitial lung disease. Clin Sci 94, 531-535 (1998).

16. Bergamaschi S, Morato E, Bazzo M, Neves F, Fialho S, Castro G, et al. Tumor markers are elevated in patients with rheumatoid arthritis and do not indicate presence of cancer. Int $\mathrm{J}$ Rheum Dis 15,179 182 (2012).

17. Fahim A, Crooks MG, Wilmot R, Campbell AP, Morice AH, Hart SP. Serum carcinoembryonic antigen correlates with severity of idiopathic pulmonary fibrosis. Respirology 17, 1247-1252 (2012).

18. Kawanami O, Ferrans VJ, Crystal RG. Structure of alveolar epithelial cells in patients with fibrotic lung disorders. Lab Invest 46, 39-53(1982).

19. Vercauteren I. M, Verleden S. E, McDonough J. E, Vandermeulen E, Ruttens D, Lammertyn E. J, et al. CYFRA 21.1 in bronchoalveolar lavage of idiopathic pulmonary fibrosis patients. Exp Lung Res 41, 459-465 (2015).

20. Tashkin D. P, Volkmann E. R, Tseng C. H, Kim H. J, Goldin J, Clements P, Furst D, Khanna D, Kleerup E, Roth M. D, Elashoff R. Relationship between quantitative radiographic assessments of interstitial lung disease and physiological and clinical features of systemic sclerosis. Ann Rheum Dis 75, 374381 (2016). 
21. Marten K, Milne D, Antoniou KM, et al. Nonspecific interstitial pneumonia in cigarette smokers: a CT study. Eur Radiol 19, 1679-1685 (2009).

22. Schwartz D. A, Merchant R. K, Helmers R. A, Gilbert S. R, Dayton C. S, Hunninghake G. W. The influence of cigarette smoking on lung function in patients with idiopathic pulmonary fibrosis. Am Rev Respir Dis 144, 504-506 (1991).

23. Rahman I, Biswas S. K, Kode A. Oxidant and antioxidant balance in the airways and airway diseases. Eur J Pharmacol 533, 222-239 (2006).

24. Van der Vaart H, Postma D. S, Timens W, ten Hacken N. H. Acute effects of cigarette smoke on inflammation and oxidative stress: a review. Thorax 59, 713-721 (2004).

25. Hodge S, Hodge G, Ahern J, Jersmann H, Holmes M, Reynolds P. N. Smoking alters alveolar macrophage recognition and phagocytic ability: implications in chronic obstructive pulmonary disease. Am J Respir Cell Mol Biol 37, 748-755 (2007).

26. Kang H. S, Cho K. W, Kwon S. S, Kim Y. H. Prognostic significance of Glasgow prognostic score in patients with acute exacerbation of idiopathic pulmonary fibrosis. Respirology 23, 206-212 (2018).

\section{Figures}

\section{Figure 1}

Flow of participants through the study

\section{Figure 2}

ROC curve of the scoring system for predicting survival of fibrosing ILD

\section{Figure 3}

Correlations between variables levels and lung function parameters in patients of fibrosing ILD at baseline 